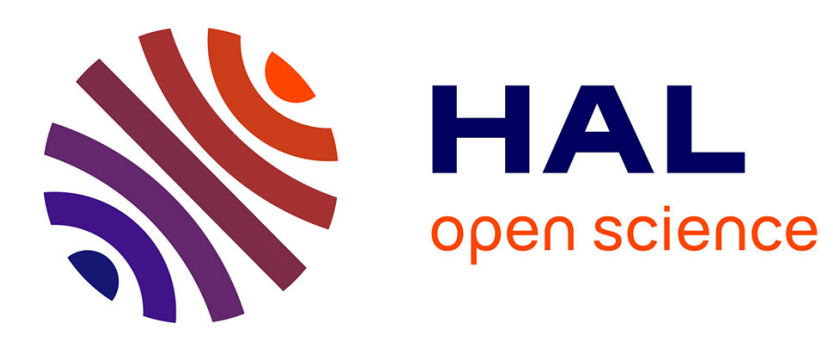

\title{
On-plate localization and mapping for an inspection robot using ultrasonic guided waves: a proof of concept
}

\author{
Cedric Pradalier, Pascal Pomarede, Jan Steckel
}

\section{To cite this version:}

Cedric Pradalier, Pascal Pomarede, Jan Steckel. On-plate localization and mapping for an inspection robot using ultrasonic guided waves: a proof of concept. 2019. hal-02290265

\section{HAL Id: hal-02290265 \\ https://hal.science/hal-02290265}

Preprint submitted on 17 Sep 2019

HAL is a multi-disciplinary open access archive for the deposit and dissemination of scientific research documents, whether they are published or not. The documents may come from teaching and research institutions in France or abroad, or from public or private research centers.
L'archive ouverte pluridisciplinaire HAL, est destinée au dépôt et à la diffusion de documents scientifiques de niveau recherche, publiés ou non, émanant des établissements d'enseignement et de recherche français ou étrangers, des laboratoires publics ou privés. 


\title{
On-plate localization and mapping for an inspection robot using ultrasonic guided waves: a proof of concept
}

\author{
Cédric Pradalier ${ }^{1}$, Pascal Pomarede ${ }^{1}$ and Jan Steckel ${ }^{2}$
}

\begin{abstract}
This paper presents a proof-of-concept for a localization and mapping system for magnetic crawlers performing inspection tasks on structures made of large metal plates. By relying on ultrasonic guided waves reflected from the plate edges, we demonstrate that it is possible to recover the plate geometry and robot trajectory to a precision comparable to the signal wavelength. The approach is tested using real acoustic signals acquired on test metal plates using lawn-mower paths and random-walks. To the contrary of related works, this paper focuses on the practical details of the localization and mapping algorithm.
\end{abstract}

\section{INTRODUCTION}

This paper aims at demonstrating the interest of using ultrasonic guided waves to support inspection robots operating on structures made of metal plates. Such structures include in particular ship outer hulls and large storage tanks, as depicted in 1. On such a structure, localization with respect to individual plates can be beneficial to precisely triangulate defects such as corrosion patches[1], [2] or even attempt acoustic tomography as in [3]. In combination with odometry and an external localization system (laser theodolite, UltraWide Band (UWB) beacons...), this would also lead to precise absolute localization of the inspection results.
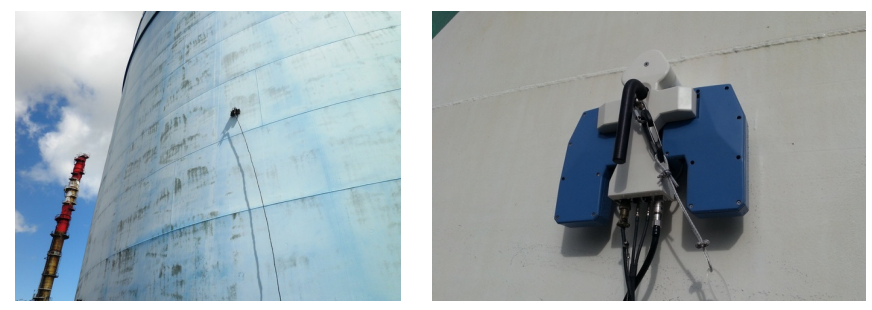

Fig. 1. Typical inspection conditions for magnetic crawlers operating on a structure assembled out of welded steel plates [Source: RoboPlanet]

On metal plates, Ultrasonic Guided Waves (UGWs) can mostly be generated using piezo-electric transducers in contact with the plate. When the relation between the frequency, the plate thickness and the wave velocity is right, these waves propagate radially around the emitter through the plate material, like ripples around a stone thrown into a pool. For metal plates relevant to inspection tasks, frequencies in the

This work was supported by the EC under grant agreement 123456

${ }^{1}$ Cédric Pradalier and Pascal Pomarede are with the GeorgiaTech Lorraine and the UMI2958 GT-CNRS in Metz, France cedric.pradalierdgeorgiatech-metz.fr

${ }^{2}$ Jan Steckel is with the CoSys-Lab, Faculty of Applied Engineering, University of Antwerp, Antwerp, Belgium and with the Flanders Make Strategic Research Center, Lommel, Belgium. jan. steckel@uantwerpen. be range of hundreds of $\mathrm{kHz}$ are typically appropriate, with wave length of the order of a few centimeters.

In this paper, we are considering short bursts of a few wave cycles emitted from a transducer mounted on a mobile platform. When encountering the plate edges, these waves are reflected normally and these reflections are measured by a transducer co-located with the emitter to be converted into electrical signals. From these signals, it is possible to identify the reflected waves and from them recover the distance to the edge. In a practical case, while working on a rectangular plate, the acquired signal may include reflections from multiple edges as well as spurious detections. These sequences of edge detections are essentially range-only measurements to a line. Our intent is to use these ranges to both reconstruct the geometry of the plate and the localization of the emitter-receiver device over time. In the robotic community, this is obviously a SLAM problem with a nontrivial data-association challenge to identify which edge has been observed at a given time. Although a number of works have considered the very similar problem of room shape reconstruction from acoustic echoes as a SLAM problem, most of them only consider simulated measurements and do not describe exhaustively their map management strategies.

In summary, the contributions of this paper are the following:

1) a demonstration of the applicability of a sparsity-based reconstruction of the arrival times of the ultrasonic reflections in the plate using an $\mathcal{L}_{1}$-regularized least squares approach;

2) a demonstration of the applicability of room reconstruction methodologies to on-plate mapping with a detailed description of the map management strategy, from initialization to landmark addition and outlier removal;

3) a proof-of-concept for the application of FastSLAM[4] to the context of on-plate localization for inspection tasks.

\section{RELATED WORKS}

a) Non-Destructive Evaluation: Ultrasound-based inspection technique is the most common tool for NonDestructive Evaluation (NDE) on metal plates. In the most common set-up, the transducers are put in transmission or in echo mode. In the first one, two transducers are aligned along the same axis, one transducer is used as an emitter and the second receives the signal after propagation through the sample. In the second mode, one transducer acts both as emitter and receiver and the signal is recorded after reflecting 
on the sample. This is typically used with high frequencies (i.e. $5 \mathrm{MHz}$ ) for thickness measurements. Alternatively, this paper consider the use of Lamb waves, a specific type of UGWs. Those waves propagate along the metal plate, potentially for large distances. In industry, they have been used to detect defects in pipelines, rails, or large structures with success [5], [6], but never deployed on autonomous mobile systems. Another technique is to use signals obtained during the inspection from different positions localize defects using acoustic tomography[1], [2]. This technique is usually used in the Structural Health Monitoring (SHM) field with sensors embedded in the structures [7], [8]. However, for such a technique to work, it is critical to know the location of the emitters and receivers with a good precision, which is a challenge for magnetic crawlers operating on an inspected structure. In general, the use of UGWs for defect detection is an active area of research in the NDE community, in particular for acoustic tomography [3] or imaging discontinuities [9]. However, most of this research assumes a known localization of the emitter and receiver with respect to the inspected plate, and to the authors' knowledge, there are no work considering that the UGWs themselves could both provide the defect detection capabilities and help inferring the plate geometry and sensor localization.

b) Echo detection: Time delay estimation is often performed using a matched filter approach, which allows high-resolution time-delay estimation under the condition that the signal bandwidth is sufficiently high, and that the auto-correlation function of the used signals is sufficiently narrow [10]. Generalizations of the matched filter to overcome spectral imperfections are often applied in situations where the signal spectrum is not flat, with the most notable example of this approach the Generalized Cross Correlation - Phase Transform. The GCC-PHAT approach is the de-facto standard in time-delay estimation, given that the signal bandwidth is sufficiently high [11]. More recently, sparsity-based methods using an $\mathcal{L}_{1}$-regularized least squares approach have surfaced as super-resolution time-delay estimation, which have been extensively applied to ultrasonic time-delay estimation [12], [13]. This approach promises the detection of highly-overlapping reflections, even under low Signal-to-Noise Ratio (SNR) conditions. In our approach, we will use this sparsity-based approach to solve the timeof-arrival estimation problem.

c) Localization and Mapping: Outside of the field of NDE, the closest problem to on-plate localization and mapping is acoustic room reconstruction. In this sub-field of acoustics and signal processing, there are a number of works trying to reconstruct the shape of a room based on acoustic echoes. Earlier works (e.g. [14]) assumed that multiple static sources where located in a polygonal room and that moving receiver was collecting data. [15] addressed the problem of a moving emitter-receiver device to reconstruct a room shape using a geometric solution. However, it used simulated data and did not assume any measurement noise. In a groundbreaking work, [16] showed that SLAM techniques could be use to estimate the shape of a convex room. This work however was only tested in simulation, and as a result assumed that all echoes were matched to room edges and that no spurious echoes were present. A series of work expanded on [16]. [17] expresses the problem as an optimization but is again tested only in simulation and does not address explicitly the ambiguous matching of measurements to room walls. In all the works above the map management strategy is not described exhaustively: in particular they do not explicit how the geometry of the room is initialized and how map edges are included when first detected or removed when proven to be outliers. On a more practical standpoint, [18] and [19] build on the article above to build a practical room reconstruction system running on a smart-phone. The latter would be used to emit sound pulses that would reflect from the room walls. In [19], a rectangular environment is assumed, with known sizes, and the focus is on localization. In [18] on the other hand, both the localization and the wall estimation are considered with only the first echo but very little information is given on the map management: when to add a new wall, how to handle outliers, etc.

In the context of SLAM, data association has always been a critical steps, as described in reference works such as [20] or [21]. FastSLAM [4] is an alternative formulation of the SLAM problem where the state is estimated by sampling and where each sample contains an estimate of the trajectory and the map collected along this trajectory. FastSLAM also allows to handle uncertain data association by including multiple samples for the various association hypotheses. This is particularly important for the problem at hand since the validity of wall hypotheses can sometimes only be estimated several steps after their initialization. To our knowledge, FastSLAM has not been used previously in the context of the acoustic room reconstruction and localization problem.

d) Summary: This work will use UGWs to build on the room reconstruction techniques within a FastSLAM framework. While moving an emitter-receiver pair on a metal plate, this will allow to recover the trajectory of the device and the geometry of the plate. To support fast and robust echo detection, we make use of an $\mathcal{L}_{1}$-regularized least squares approach to signal-delay estimation using a sparse signal model. This approach allows very accurate time-delay estimation of multiple overlapping reflections to be performed, even in low SNR conditions. The next section will describe how these components combine together to demonstrate the feasibility of using UGW for on-plate localization and plate geometry inference.

\section{MeThODOLOGY}

\section{A. Notations and assumptions}

In this paper we are considering a mobile unit transporting an acoustic emitter-receiver pair on a metal plate. At every time step $k$, the emitter sends an acoustic pulse $s_{b}^{k}(t)$. Under mild assumptions of the acoustic properties of the ultrasonic probing system, we can model the received transducer signal $s_{r}^{k}(t)$ as a linear system through convolution:

$$
s_{r}(t)=x(t) * s_{b}(t)
$$


with $x(t)$ the environments impulse response and $s_{r}(t)$ hopefully containing information about reflections on the plate edges. The superscript $k$ is omitted when unambiguous.

We assume that the plate is an homogeneous material (steel, aluminium,...) of a constant thickness. We assume the plate to be a convex polygon, but we do not assume it to be rectangular, even though this is to be expected in any industrial case. Because the edges are linear, we will also assume that only the orthogonal reflection of the signal is picked up by the receiver. Any other reflection may lead to secondary echoes after bouncing from several edges but these are neglected in our work.

Out of the reflected signal $s_{r}^{k}(t)$, we will denote as $\left\{r_{i}(k), i=1 \ldots n(k)\right\}$ the set of detected ranges to the plate edges, assuming that not all the edges are detected all the time and that spurious detection may be included in the list.

Additionally, because we assume the transducers are carried by a mobile device on the plate, we assume that some level of odometry is available to estimate the displacement between measurements. Because the inspection crawlers we are considering are moving on vertical structures (boat hulls, storage tanks), they can easily embed an accelerometer from which the crawler heading with respect to gravity can be observed with a good enough precision. Also, by construction, to avoid falling from the inspected structures, these crawlers have to have an extremely good adherence and incur very little slippage. We can hence assume the odometry locally very precise. These two assumptions (known heading and very precise odometry) are an important distinction with respect to the room reconstruction works.

In the next section we will describe first how these echoes can be robustly extracted from the signal and then how they can be integrated with the proprioceptive measurements into a localization and mapping framework.

\section{B. Echo detection}

One of the key enabling technologies for the on-plate SLAM approach is the robust and accurate detection of ultrasonic echoes in the metallic plate. Due to the physics of the sound propagation in the metallic plate, only signals with limited bandwidth can be used for probing the underlying material. This limited bandwidth constraint complicates accurate time-of-arrival estimation for the reflections in the medium, removing classical signal processing techniques such as matched filtering from the set of applicable techniques. We apply a super-resolution technique based on $\mathcal{L}_{1}$-regularized least-squares and the concept of sparsity to solve this estimation problem. The underlying hypothesis of this approach is that most of the metal plate does not reflect the ultrasonic signals, and that only discontinuities in the medium cause reflections to occur. In that case, the environment has only a limited number of reflections, and can be considered sparse.

Under the sparsity assumption of the environment, we can consider $x(t)$ from eq. 1 to be sparse. After discretization of the problem, this allows us to cast the following $\mathcal{L}_{1}$ - regularized least squares problem:

$$
\min _{\mathbf{x}}\left|\mathbf{D} \cdot \mathbf{x}-\mathbf{s}_{\mathbf{r}}\right|_{2}^{2}+\lambda \cdot|\mathbf{x}|_{1}
$$

with $\mathbf{x}$ the discretized vector-representation of the impulse response $x(t)$ of size $\left[n_{t} \times 1\right], \mathbf{s}_{\mathbf{r}}$ the discretized received acoustic signal of size $\left[n_{t} \times 1\right]$, where $n_{t}$ is the number of time-samples used in the discretization process. The vector norms $|a|_{p}$ denote the $\mathcal{L}_{p}$-norm of vector-object $a$, with the well-known fact that the $\mathcal{L}_{1}$-norm promotes sparsity of the least-squares solution. The matrix $D$ is called a dictionary matrix of size $\left[n_{t} \times n_{t}\right]$, containing time-shifted copies of the emitted echo signal $\mathbf{s}_{\mathbf{b}}(\mathbf{t})$ :

$$
\mathbf{D}=\left[\begin{array}{ccccc}
\mathbf{s}_{\mathbf{b}}(\mathbf{t}) & 0 & 0 & \ldots & 0 \\
0 & \mathbf{s}_{\mathbf{b}}(\mathbf{t}) & 0 & \ldots & 0 \\
0 & 0 & \mathbf{s}_{\mathbf{b}}(\mathbf{t}) & \ldots & 0 \\
\ldots & \ldots & \ldots & \ldots & \ldots \\
0 & 0 & 0 & \ldots & \mathbf{s}_{\mathbf{b}}(\mathbf{t})
\end{array}\right]
$$

This minimization problem is a convex problem which can easily be solved using open-source toolboxes such as the Matlab CVX toolbox [22].

\section{From echoes to edge hypotheses}

A single echo $r_{i}(k)$ provides information about the distance to an object. As will be described later, this information is useful to refine the parameters of the edge the echo reflected from. However, this is too ambiguous to initialize a new edge: it could be any tangent to the circle centered on the current pose with radius $r_{i}(k)$.

In [16] on the other hand, the authors show that 2 echoes taken at different known position are sufficient to define two line hypotheses: there are only two lines tangent to the two circles defined by these radius and centered on the known poses. A third echo can be used to disambiguate between the two lines if the trajectory is not parallel to the line. Beside requiring three perception steps and the corresponding delay, using three echoes requires considering all the $O\left(n^{3}\right) 3$ tuples.

Instead, we decided to use only the last two echoes and deal with the multiple hypotheses in a later step. There are several arguments for this choice: first the disambiguation with 3 echoes is only useful when the path is not parallel to the edge, which is actually a very common case in a practical deployment where the robot use its accelerometer to realize vertical or horizontal transects. Second, even on a non-parallel path, a clear disambiguation require a large translation between the second and third echo to compensate for the perception noise. Last, when moving on a direction normal to the edge, two measurements are sufficient for a unique solution. Both cases are illustrated in fig. 2.

Because of our combinatorial generation of edge hypotheses, a method is required to identify inconsistent edges and remove them. To this end, we take advantage of the hypothesis that only orthogonal reflections are reaching the receiver. Hence, with the assumption of a convex plate, if we consider two edges $L_{1}$ and $L_{2}$ observed from pose $P$ and $P_{i}$ the projection of $P$ on $L_{i}$, then $L_{2}$ cannot intersect the 


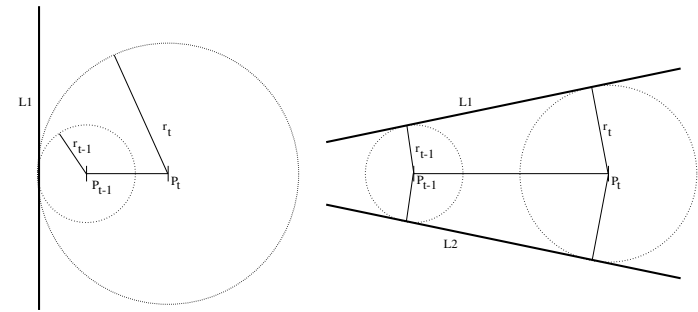

Fig. 2. Line hypotheses generated from two measurements at position $P_{t-1}$ and $P_{t}$. Left: the robot moves away from the edge and generate a single hypothesis, right: generic case leading to two hypotheses.

segment $\left[P P_{1}\right]$ and $L_{1}$ cannot intersect the segment $\left[P P_{2}\right]$. If one of this condition is true, then both edges cannot exist simultaneously on a convex plate because observing one edge would require the ultrasonic waves to cross the other one, which is not physically possible. In the following, this test will be denoted as the consistency check between two edge hypotheses.

\section{FastSLAM integration}

FastSLAM[4] is a solution to the simultaneous localization and mapping problem which is particularly relevant in the context of uncertain data association. In essence, FastSLAM relies on a particle filter in the localization space and every particle holds an hypothesis of the map that can be inferred from the trajectory of this particle. In order to integrate FastSLAM into our localization and plate geometry inference problem, this section will define the particle state, the chosen map representation, the particle initialization strategy, how the internal maps are updated and the evaluation of a particle based on the measured echoes.

1) FastSLAM state: Because of our assumption of a known orientation, we only consider the sensor coordinates $(x, y)$ on the plate as system state. Orientation could be added to the state without significant changes to the framework.

As a map representation, we decided to use a list of infinite lines represented by an angle and offset. Several options were considered and ultimately rejected. Inspired from traditional occupancy grids[23], we first considered storing a grid map where every cell would represent either the likelihood of being on an edge or the likelihood of being inside the plate. Both representations work well in a mapping only framework but in a SLAM context they lack robustness. Using the edge likelihood presents a challenge for removing outliers and artifacts. Using the likelihood of being inside the plate works well when only using the first echo (i.e. the closest edge) but does not scale to using echoes from multiple edges. Using a parameterization as an oriented rectangle was also evaluated but rejected because first, it only works for rectangles and second, the estimator tended to forget about one side of the rectangle when only the other one was observed for too long. In the end, estimating independent lines offers more flexibility and provides sufficient information about the plate geometry. In practice, a line is represented by a pair $(\theta, b)$ that define the line equation:

$$
\cos (\theta) \cdot x+\sin (\theta) \cdot y+b=0
$$

A particle $P_{i}$ can then be described as a trajectory hypothesis associated with an estimated map defined as a set of lines:

$$
P_{i}=\left[\left(x_{i}, y_{i}\right)_{i=1 \ldots k},\left\{\left(\theta_{j}, b_{j}\right), j=1 \ldots n_{i}\right\}\right]
$$

2) FastSLAM initialization: Most of the earlier papers[16], [17] on room reconstruction do not discuss the initialization of the Bayesian filter, which is an important element of a practical implementation. In our case, we rely on the edge hypotheses generated from section III-C. After waiting for the second set of range measurements, we estimate a number of edge hypotheses from which we extract maximally consistent sets. These sets are built using a dynamic programming approach inspired from JCBB/JCDA[20] which will be omitted here for the sake of page limits. As a result, every particle is initialized by sampling around the zero position and randomly selecting a consistent set of edges from the maximally consistent sets. Including all the edge hypotheses in all the particles would be a viable alternative given the outlier removal decision described below. It would however add more ambiguity than necessary in the estimation.

3) Particle evaluation: Given $\left\{r_{i}(k), i=1 \ldots n(k)\right\}$ the set of echoes measured at time $k$, we evaluate a particle based on its ability to explain the measurements. For a line $L_{i, j}=\left(\theta_{i, j}, b_{i, j}\right)$ in particle $P_{i}$, the expected measured range is

$$
d_{i, j}=\left|\cos \left(\theta_{i, j}\right) \cdot x_{i}+\sin \left(\theta_{i, j}\right) \cdot y_{i}+b_{i, j}\right|
$$

From this range, the likelihood of measurement $r_{i}(k)$ given $L_{i, j}$ is expected to follow a Gaussian distribution centered on $d_{i, j}$, with a standard deviation consistent with the echo detection uncertainty. If the highest likelihood over all the lines is lower than a threshold, then the measurement is considered "unexplained" and allocated a low probability $P_{0}$.

For a complete set of echoes, the evaluation of a particle will then be the product of the likelihood of the $n(k)$ likelihoods of the independent measurements:

$$
\mathcal{L}\left(P_{i}\right)=\prod_{i=1}^{n(k)} \max \left(P_{0}, \max _{j}\left(P\left(r_{i}(k) \mid P_{i} L_{i, j}\right)\right)\right)
$$

In this formulation, the product has $n(k)$ terms which make the evaluation of different particles comparable. Performing the product over the set of lines of each particle would result in a varying number of terms, which is incompatible with importance sampling.

Note that for the purpose of the map update, if a measurement is considered explained, the index of the line leading to highest likelihood is recorded as $j_{k, i}^{\star}$. The list of unexplained measurement is also stored to create additional line hypotheses. 
4) Map update: The map update stage has three purposes, first for "explained" measurements, the associated line needs to be updated to account for the new piece of information. Second, the "unexplained" measurements are used to create additional line hypotheses. Third, consistency checks are used to eliminate line hypotheses which are no longer supported by the observations.

a) Line update: Knowing a measurement $r_{i}(k)$ and its associated line in particle $P_{i}, L_{i, j_{k, i}^{\star}}$, one needs to update the corresponding $\left(\theta_{j^{\star}}, b_{j^{\star}}\right)$. The definition of $d_{i, j}$ suggest the use of an Extended Kalman Filter. Even though such a filter is feasible, the individual measurements can often be explained equivalently by changing $\theta_{j^{\star}}$ or $b_{j^{\star}}$, which prevents a precise convergence of the filter. To sidestep this issue, an alternative is to keep a record of the sensor poses and ranges associated with this line and use a non-linear minimization of the following cost function:

$$
\begin{aligned}
& C_{j^{\star}}(\theta, b)= \\
& \quad \sum_{s=1}^{k}\left[\left(\cos (\theta) \cdot x_{i}(s)+\sin (\theta) \cdot y_{i}(s)+b\right)^{2}-r(s)^{2}\right]
\end{aligned}
$$

Although much more expensive, this approach converges without bias even when the measurements are taken on a monotonic walk along the edge.

b) New lines: For unexplained measurements, we need to combine them with the measurements from the previous time step to create edge hypotheses (sec. III-C). Every pair made of one previous measurement and a new unexplained measurement is used to generate one or two edge hypotheses. All the previous measurements are used for additional robustness against incorrectly associated measurements at the previous time step. All these hypotheses are added to the line set of the current particles.

c) Map clean-up: In a final stage of the map update, all the lines in a particle line set are checked for pairwise consistency. If two lines are deemed inconsistent and one of them has been observed (i.e. associated with a measurement) significantly more than the other since its creation, then the least observed line is marked for deletion. In our implementation, this criterion is defined as being observed two additional times. In this stage we also mark for deletion lines that were not re-observed enough since their creation. For instance, we delete a line that is more than 10 stepold but has been observed less than 3 times. The simple delayed outlier removal described in this paragraph is what makes possible the generation of many edge hypotheses from the set of detected echoes. This is critical since many of these hypotheses cannot be ruled out until the robot moves significantly and changes direction. In comparison, in [16] the authors made the hypotheses of a random walk of the agent, which is a much more informative path, but also much less realistic for a robotic crawler.

\section{RESULTS}

\section{A. Experimental setup}

At the time of this writing, the experimental setup is not yet integrated on a robotic crawler. Instead, in order to test our framework in a setup as close as possible from reality, we used a pair of emitter-receiver piezo-electric transducer on two different aluminium plates (plate 1: 470x470x5mm and plate 2: $600 \times 450 \times 6 \mathrm{~mm}$ ) and moved them by hand on the vertices of a regular grid with 30 to $40 \mathrm{~mm}$ spacing. At every position, the response to 10 ultrasonic scans were averaged and recorded with their acquisition position. A scan in this context is the emission of two periods of a $100 \mathrm{kHz}$ sine wave and the recording of $400 \mu \mathrm{s}$ of analog signal at 1 Msample/second.

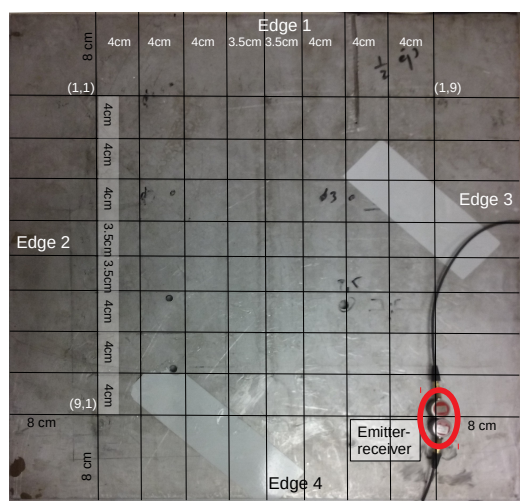

Fig. 3. Experimental setup for metal plate 1. The emitter and receiver transducer can be seen on the bottom right of the figure.

To simulate a sweep of a plate by a robotic crawler, the corresponding sequence of measurements is selected from the database and presented to the SLAM framework, with the theoretic displacement between grid cells used as odometry. We believe that, apart from the size of the plates which is smaller than real ones, this setup is a correct representation of a real system in terms of signal quality and overall input precision.

\section{B. Echo detection}

Figure 4 illustrates the echo detection process. Panel a) shows the emitted signal, which is a 2 -cycle burst at $100 \mathrm{kHz}$. The reflected signal from the plate structure can be seen in panel b). It shows the pickup of the emission in the beginning of the signal, and a reflection due to coupling mismatches. Then, a series of echoes is apparent. Using the $\mathcal{L}_{1}$-based approach, the impulse response can be reconstructed (see panel $b$, orange trace). The method reconstructs the major echo components of the impulse response, while ensuring that the solution is sparse. Panel c) shows the reconstruction error. The fact that this residual is not zero can be explained by the fact that the direction-dependent filtering of the transducer is not taken into account during the reconstruction phase. However, as the remaining experiments show, this crude approach to the echo detection process is sufficient for the plate-estimation algorithm. 

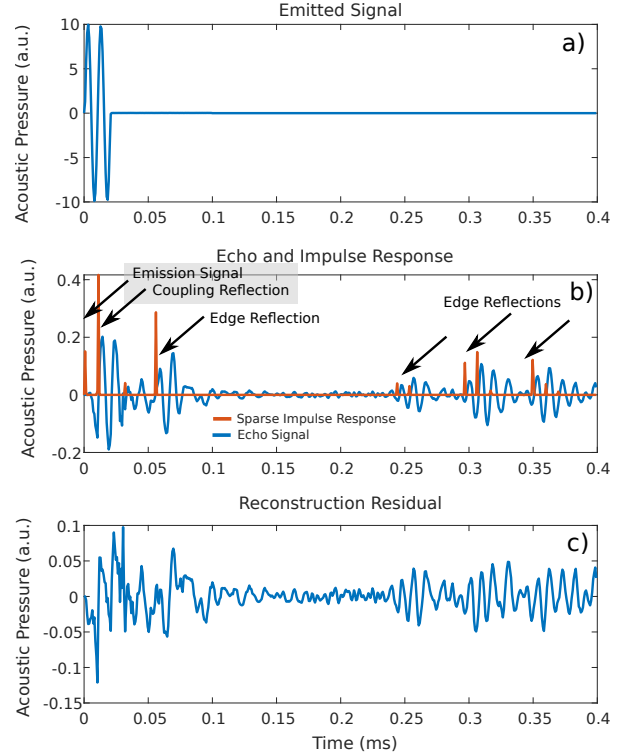

Fig. 4. Illustration of the echo detection process. Panel a) shows the emitted signal. Panel b) shows the echo signal (blue trace) and the reconstructed impulse response (orange trace). Panel c) shows the reconstruction residual. The remaining energy in that residual can be explained by the mismatch in the used signal model $D$ and the real transducer.

\section{Edge hypotheses}

Fig. 5 gives an example of computed edges from the combination of the two first measurements on plate 2. As can be observed, the number of edge hypotheses is relatively large but a few of them are definitely good estimates of the real top and bottom edges. At this position, lateral edges are not observed correctly. The level of uncertainty in the putative edges is what calls for the FastSLAM framework and the delayed decision the validity of the edges.

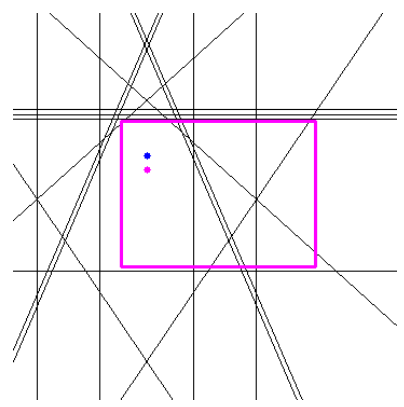

\begin{tabular}{|c|c|}
\hline$r(1):$ & 0.1151, \\
& 0.1297, \\
& 0.1436, \\
& 0.3406, \\
& 0.3557 \\
\hline \hline$r(2):$ & 0.1454, \\
& 0.1594, \\
& 0.1788, \\
& 0.1933, \\
& 0.3163, \\
& 0.3333, \\
& 0.4484 \\
\hline
\end{tabular}

Fig. 5. Edge hypotheses generated on plate 2 from detected echoes $r(1)$ (blue dot) and $r(2)$ (purple dot), without consistency check. The purple rectangle is the outline of the true plate.

\section{Mapping performance}

Figure 6 shows one anecdotal example of a mapping run on plate 2 by displaying the highest-ranked particle (out of 16 in this case), its line set and its trajectory. The reconstructed path is clearly visible and the stability of the estimate can be observed when performing a second sweep of the path (iterations 225 and 239). The convergence of the estimated lines to the true plate outline can also be observed, with a final error in the order of $2 \mathrm{~cm}$ on the line offsets $b$ (see eq. 4 ). Even though this run has a lower precision than the average case, $2 \mathrm{~cm}$ is still a very acceptable precision given that the sensors are positioned by hand and that the wave length in this plate is approximately $3 \mathrm{~cm}$.

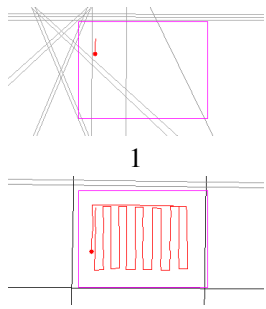

124

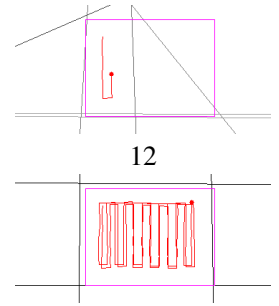

225

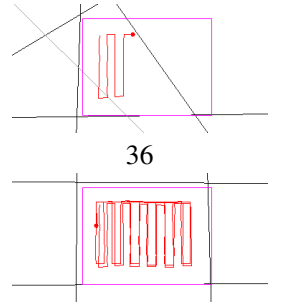

239
Fig. 6. Evolution of the map representation for the highest-ranked particle at step 1, 12, 36, 124, 225 and 239. The purple frame represent the outline of the true plate. The red dot is the current estimated sensor pose and the red line is the history of its estimated trajectory. The darkness of the line is proportional to the number of times they have been observed.

As a final evaluation, we evaluated the precision and repeatability of our approach through 100 repetitions in three scenarios. Scenario 1 and 2 consisted of a lawn-mover path through the plate 1 and 2 . The length of the sweeping path being 81 steps and 108 steps respectively. In a third scenario, we used plate 1 and simulated localization and plate geometry estimation over a random walk. In the three cases, the real measurements described above were used. However, a single database of measurements was used for every repetition. The table below presents a summary of the resulting precision at different steps in the process. It is clear that the initial estimates after observing only half of the plate are still very uncertain for all the scenarios, but at the end of the simulation all lines from plate 1 are estimated with a precision consistent with the signal wavelength. The poorer performance on plate 2 are due to the somewhat larger size of the plate and to the presence of artificial defects that are acting as reflectors and creating detection artifacts.

\begin{tabular}{|l|c|c|}
\hline Scenario & Angle error [rad] & Offset error [m] \\
\hline Scenario 1, step 50 & $0.017 \pm 0.057$ & $0.004 \pm 0.032$ \\
\hline Scenario 2, step 50 & $-0.029 \pm 0.154$ & $-0.061 \pm 0.199$ \\
\hline Scenario 3, step 50 & $-0.008 \pm 0.084$ & $-0.031 \pm 0.133$ \\
\hline \hline Scenario 1, step 239 & $0.005 \pm 0.020$ & $0.002 \pm 0.010$ \\
\hline Scenario 2, step 239 & $-0.007 \pm 0.091$ & $-0.015 \pm 0.126$ \\
\hline Scenario 3, step 239 & $-0.001 \pm 0.040$ & $0.002 \pm 0.045$ \\
\hline
\end{tabular}

\section{Conclusions}

This paper presented a proof-of-concept for a localization and plate geometry inference framework for a magnetic crawler performing inspection of structures assembled out of metal plates. The results show that there is enough information in the reflected signals to achieve a good localization and mapping precision as long as a sufficient coverage of the plate is performed. The next steps will be to embed this framework on a robotic platform as presented in fig. 1, test on larger plate, improve the overall system robustness and consider an active sensing strategy to recover the plate geometry faster and even more reliably. 


\section{REFERENCES}

[1] T. Druet, B. Chapuis, M. Jules, G. Laffont, and E. Moulin, "Passive SHM system for corrosion detection by guided wave tomography," in Sensors, Algorithms and Applications for Structural Health Monitoring. Springer, 2018.

[2] P. Huthwaite and F. Simonetti, "High-resolution guided wave tomography," Wave Motion, 2013.

[3] W. Cailly and H. Walaszek, "Three dimensional ultrasonic imaging of mechanical components by inversion," in 7th edition of the International Symposium on AirCraft Materials, 2018.

[4] M. Montemerlo, S. Thrun, D. Koller, B. Wegbreit, et al., "Fastslam: A factored solution to the simultaneous localization and mapping problem," Aaai/iaai, vol. 593598, 2002.

[5] Z. Su, L. Ye, and Y. Lu, "Guided lamb waves for identification of damage in composite structures?: A review," J. Sound Vib, 2006.

[6] P. Cawley and D. Alleyne, "The use of lamb waves for the long range inspection of large structures," Ultrasonics, 1996.

[7] E. Larose, O. I. Lobkis, and R. L. Weaver, "Passive correlation imaging of a buried scatterer," J. Acoust. Soc. Am, 2006.

[8] L. Chehami, E. Moulin, de J. Rosny, C. Prada, O. B. Matar, F. Benmeddour, and J. Assaad, "Detection and localization of a defect in a reverberant plate using acoustic field correlation," Journal of Applied Physics, 2014.

[9] H. Walaszek, F. Zhang, and M. Castaing, "New testing method for the rapid an flexible ultrasonic imaging of large metal structures of composites by combining guided wave and matrix phased array technologies," in 11th European Conference on Non-Destructive Testing, 2014.

[10] G. Turin, "An introduction to matched filters," Information Theory, IRE Transactions on, vol. 6, no. 3, pp. 311-329, 1960. [Online]. Available: http://ieeexplore.ieee.org/xpls/abs $\left\{\_\right\}$all. jsp?arnumber $=1057571$

[11] C. Knapp and G. Carter, "The generalized correlation method for estimation of time delay," Acoustics, Speech and Signal Processing, ..., vol. 2, pp. 320-327, 1976. [Online]. Available: http://ieeexplore.ieee.org/xpls/abs $\{-\}$ all.jsp?arnumber $=1162830$

[12] J. Steckel and H. Peremans, "Sparse decomposition of in-air sonar images for object localization," in Proceedings of IEEE Sensors, vol. 2014-Decem, no. December, 2014.

[13] B. Fontaine and H. Peremans, "Determining biosonar images using sparse representations." The Journal of the Acoustical Society of America, vol. 125, no. 5, pp. 3052-9, may 2009.

[14] D. Salvati, C. Drioli, and G. L. Foresti, "Sound source and microphone localization from acoustic impulse responses," IEEE Signal Processing Letters, vol. 23, no. 10, pp. 1459-1463, 2016.

[15] F. Peng, T. Wang, and B. Chen, "Room shape reconstruction with a single mobile acoustic sensor," in 2015 IEEE Global Conference on Signal and Information Processing (GlobalSIP). IEEE, 2015, pp. $1116-1120$.

[16] M. Kreković, I. Dokmanić, and M. Vetterli, "Echoslam: Simultaneous localization and mapping with acoustic echoes," in 2016 IEEE International Conference on Acoustics, Speech and Signal Processing (ICASSP). Ieee, 2016, pp. 11-15.

[17] M. Krekovic, I. Dokmanic, and M. Vetterli, "Look, no beacons! optimal all-in-one echoslam," arXiv preprint arXiv:1608.08753, 2016.

[18] S. Pradhan, G. Baig, W. Mao, L. Qiu, G. Chen, and B. Yang, "Smartphone-based acoustic indoor space mapping," Proceedings of the ACM on Interactive, Mobile, Wearable and Ubiquitous Technologies, vol. 2, no. 2, p. 75, 2018.

[19] X. Song, M. Wang, H. Qiu, and L. Luo, "Indoor pedestrian selfpositioning based on image acoustic source impulse using a sensor-rich smartphone," Sensors, vol. 18, no. 12, p. 4143, 2018.

[20] J. Neira and J. D. Tardós, "Data association in stochastic mapping using the joint compatibility test," IEEE Transactions on robotics and automation, vol. 17, no. 6, pp. 890-897, 2001.

[21] T. Bailey, E. M. Nebot, J. Rosenblatt, and H. F. Durrant-Whyte, "Data association for mobile robot navigation: A graph theoretic approach," in Proceedings 2000 ICRA. Millennium Conference. IEEE International Conference on Robotics and Automation. Symposia Proceedings (Cat. No. O0CH37065), vol. 3. IEEE, 2000, pp. 2512-2517.

[22] M. Grant, S. Boyd, and Y. Ye, "Cvx: Matlab software for disciplined convex programming," 2008.

[23] A. Elfes, "Using occupancy grids for mobile robot perception and navigation," Computer, vol. 22, no. 6, pp. 46-57, 1989. 